\title{
Effects of Insulin and Dietary Myoinositol on Impaired Peripheral Motor Nerve Conduction Velocity in Acute Streptozotocin Diabetes
}

\author{
Douglas A. Greene, Pacifico V. De Jesus, Jr., and Albert I. Winegrad \\ From the George S. Cox Medical Research Institute, Department of Medicine, \\ and the Department of Neurology, University of Pennsylvania School of \\ Medicine, Philadelphia, Pennsylvania 19104
}

A в S T R A C T The factors influencing the development of impaired sciatic motor nerve conduction velocity (MNCV) in acute experimental diabetes were examined. Decreased MNCV developed by the 14th day after streptozotocin administration but only in rats which became hyperglycemic. Insulin treatment, begun on day 3 , failed to prevent impaired $\mathrm{MNCV}$ in diabetic rats in which improved or normal weight gain and a decreased degree of hyperglycemia was induced. However, insulin treatment prevented the development of impaired $\mathrm{MNCV}$ in a group of diabetic rats in which the tail vein plasma glucose concentration was never found to exceed 160 $\mathrm{mg} / \mathrm{dl}$ during days 6 through 14, and in which the mean $\pm S E M$ of the average plasma glucose concentration for each animal during the same period was $75 \pm 18$ $\mathrm{mg} / \mathrm{dl}$. In normal rats fed diets containing $0.011 \%$ or $0.069 \%$ free myoinositol (a presumably normal range), sciatic nerve free myoinositol concentrations were 90 and 60 -fold higher than those in plasma. On these diets the development of impaired MNCV in the diabetics was associated with a decrease in nerve free myoinositol as compared with nerves from normals fed the same diet, despite similar plasma levels in the normals and diabetics. Plasma and nerve free myoinositol increased with increasing dietary myoinositol content in both normals and diabetics, and nerve myoinositol content could be acutely increased by an i.p. myoinositol load. By supplementing the diets with $1.0 \%$ myoinositol, the difference in nerve myoinositol in normal and diabetic rats on day 14 was abolished; on this diet the development of impaired MNCV in the diabetics was moder-

Dr. Winegrad is the recipient of a Research Career Development Award from the National Institutes of General Medical Studies (GM 6405).

Received for publication 4 December 1974 and in revised form 17 February 1975. ated or totally prevented, despite persistent hyperglycemia and elevated nerve sorbitol and fructose concentrations. Insulin treatment that prevented impaired MNCV prevented a decrease in nerve myoinositol in diabetics. These studies suggest that insulin deficiency, and possibly hyperglycemia, are primary factors in the development of impaired MNCV in acute experimental diabetes. However, the development of impaired $\mathrm{MNCV}$ appears to be related in some manner to a derangement in the regulation of nerve free myoinositol content, which appears to be subject to modification by increases in plasma myoinositol concentration over a critical range.

\section{INTRODUCTION}

The development of the most common form of diabetic neuropathy, distal symmetrical polyneuropathy, is thought to be conditioned by some chronic metabolic disturbance, for recent pathological studies seem to exclude occlusive vascular disease as a primary causative factor (1-5). However, the importance of insulin deticiency in the pathogenesis of diabetic polyneuropathy is still disputed because of ambiguities in the data concerning the relationship between the degree of antecedent "diabetic control" and the development of this syndrome, and the response to insulin treatment in patients with overt polyneuropathy (6). Patients with diabetic polyneuropathy exhibit decreased peripheral motor and sensory nerve conduction velocities, and similar alterations have been found in long-standing diabetics who have no evidence of polyneuropathy $(3,7-9)$. In both of these instances, the decreased nerve conduction velocities are associated with lesions in peripheral nerve biopies, which are more marked in the patients with overt polyneuropathy (3). These lesions include loss of myelinated 
axons, evidence of segmental demyelination and remyelination, and in some instances Schwann cell proliferation $(1,3)$. Recently it has been reported that newly diagnosed juvenile diabetics frequently have decreased peripheral motor and sensory nerve conduction velocities, despite a normal neurological examination and the absence of symptoms (10). The pathological lesions, if any, present in the peripheral nerves of newly diagnosed juvenile diabetics have not been identified. The initiation of insulin treatment appears to result in improvement in peripheral nerve conduction velocity in juvenile diabetics (10); however, there is evidence that nerve conduction velocity tends to decrease with increasing duration of disease in a manner that cannot be attributed to the effects of age (11). There is, as yet, no clear evidence that the progression of impaired nerve conduction velocity in juvenile diabetics correlates with the progression of the lesions found in association with overt polyneuropathy. However, there are, at the same time, no compelling reasons to dismiss the possibility that the alterations in peripheral nervous function found in newly diagnosed juvenile diabetics may reflect a metabolic derangement that contributes to the later development of lesions and overt polyneuropathy in some longstanding diabetics.

Efforts to develop an appropriate animal model for overt diabetic polyneuropathy have thus far been unsuccessful; however, in 1964 Eliasson found that the induction of experimental diabetes in rats by pancreatectomy or alloxan administration resulted in impaired sciatic motor and sensory nerve conduction velocities within 2 wk (12). This impairment did not occur in rats that failed to become hyperglycemic after exposure to alloxan. However, Eliasson was unable to prevent the development of impaired nerve conduction velocities by insulin treatment, or to affect it by the addition of insulin to the isolated nerve in vitro (12). Although there have been two reports that impaired nerve conduction velocity in rats with experimental diabetes can be improved by insulin treatment $(13,14)$, it has not been possible to prevent its development, and Sharma and Thomas recently concluded that "the influence of insulin on conduction velocity in diabetic animals is so far uncertain" (15).

The studies that form the basis of this report are concerned with the factors that influence the development of impaired sciatic motor nerve conduction velocity $(\mathrm{MNCV})^{1}$ in rats with acute streptozotocin diabetes. These studies provide evidence of a definite relationship between an impaired insulin secretory mechanism, as manifested by hyperglycemia, and the development of impaired MNCV. In addition, they demonstrate that

${ }^{1}$ Abbrcviations used in this paper: MI, myoinositol; $\mathrm{MNCV}$, motor nerve conduction velocity. acute experimental diabetes results in an impaired ability to maintain normal peripheral nerve free myoinositol (MI) concentrations, which appears to be related to the development of impaired MNCV. This alteration in nerve free MI content can be prevented by insulin treatment adequate to prevent impaired MNCV. Most mammalian cells contain free MI concentrations much higher than those in plasma; however, the mechanisms responsible for this concentration gradient have not been clarified in peripheral nerve, or in most tissues $(16,17)$. Both human and experimental diabetes are known to result in marked increases in urinary free MI excretion as a consequence of the effects of high glucose concentration on the tubular reabsorption of free MI (18). However, little significance has been attributed to these observations, since there is no well-established requirement for exogenous free $\mathrm{MI}$ in normal rats or man $(19,20)$. We found that the free MI concentration in the peripheral nerves of normal and acutely diabetic rats is subject to modification by alterations in dietary free MI content and plasma free MI concentration. Pharmacological increases in dietary free MI content over a critical range were shown to result in similar elevated levels of free MI in the peripheral nerves of diabetic and normal rats and to prevent or modify the development of impaired $\mathrm{MNCV}$ in acute experimental diabetes, despite severe persistent hyperglycemia.

These observations suggest that acute experimental diabetes induces an alteration in free MI metabolism in peripheral nerve that is related to the development of impaired MNCV, but potentially subject to modification by factors influencing the plasma free MI concentration, such as dietary free MI content and renal disease.

\section{METHODS}

Male, Wistar rats, initial wt 145-155 g, were fed ad libitum throughout the 14-day study. Body weight was determined every other day, except in the insulin-treated diabetic rats, in which it was determined daily. The standard diet consisted of pellets composed of $18 \%$ vitamin-free casein, $68 \%$ sucrose, $10 \%$ vegetable oil, $4 \%$ inorganic salts, all known rat vitamin requirements, and $0.011 \%$ free MI (Nutritional Biochemicals Corp., Cleveland, Ohio). The same supplier provided pellets prepared from the standard diet supplemented with $1 \%$ or $3 \%$ free MI by weight. Wayne rat blocks (Allied Mills, Inc., Chicago, Ill.) were ground in a hand mill to provide the chow diet. In specific instances the ground chow was mixed with $1 \%$ free MI (Sigma Chemical Co., St. Louis, Mo.) by weight.

Experimental diabetes was induced by injecting $70 \mathrm{mg} / \mathrm{kg}$ of streptozotocin (Upjohn Company, Kalamazoo, Mich.) in $0.10 \mathrm{ml}$ of $0.01 \mathrm{M}$ citrate buffer, $\mathrm{pH} 5.5$, into a tail vein of rats that had been fasted overnight. Nonfasting plasma glucose concentrations in excess of $300 \mathrm{mg} / \mathrm{dl}$ at both 24 and $48 \mathrm{~h}$ after streptozotocin administration were required for the diagnosis of experimental diabetes. Rats that failed to become hyperglycemic were maintained as streptozotocin controls, but this was an infrequent occurrence. 


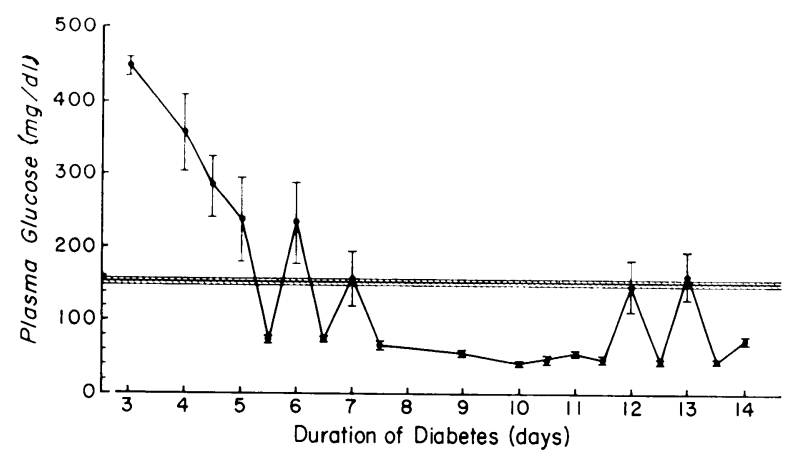

Figure 1 Plasma glucose concentrations observed in tail vein samples from the insulin-treated streptozotocin diabetic rats in group 3 ; the values depicted are the means \pm SEM for the 17 animals that survived until day 14. The rats received Lente insulin twice a day in adjusted doses, as detailed in Methods, beginning on day 3 after streptozotocin administration. The horizontal lines and striped area represent the mean \pm SEM tail vein plasma glucose concentration found on the morning of day 14 in 11 normal rats fed the same standard diet.

Insulin treatment of diabetic rats was begun on day 3 after streptozotocin administration. Three groups of insulintreated diabetics were treated in different fashions. Group 1 received single daily subcutaneous injections of Ultralente insulin (Eli Lilly and Company, Indianapolis, Ind.) initially in doses of $8 \mathrm{U}$, which were increased to $10 \mathrm{U}$ from day 8 after streptozotocin administration to the completion of the study on day 14 . Group 2 received twice daily injections of Lente insulin (Lilly); the initial doses were $6 \mathrm{U}$ between 9 and 10 a.m. and $12 \mathrm{U}$ between 6 and 7 p.m.; the doses were adjusted individually on the basis of plasma glucose determinations and during the period from day 10 to 14 they ranged from 4 to $8 \mathrm{U}$ in the morning and 14-20 U in the evening. Group 3 received twice daily subcutaneous injections of Lente insulin. Plasma glucose was determined twice daily, in the morning and in the late afternoon, on most days to permit adjustment of the insulin dosage. The initial doses were $3.0 \mathrm{U} / 100 \mathrm{~g}$ wt in the morning and 10 $\mathrm{U} / 100 \mathrm{~g}$ wt in the evening. The average insulin dosage increased until day 9 when it was $4.0 \mathrm{U} / 100 \mathrm{~g} \mathrm{wt}$ in the morning and $14 \mathrm{U} / 100 \mathrm{~g}$ in the evening. Because of the development of hypoglycemia, which caused the death of 7 of the 24 rats in group 3, the insulin dosage was reduced on days 11-14 to an average of $2 \mathrm{U} / 100 \mathrm{~g}$ wt in the morning and $5.0 \mathrm{U} / 100 \mathrm{~g}$ wt in the evening. The mean plasma glucose values in these animals are shown in Fig. 1. Plasma glucose was determined in $0.20-\mathrm{ml}$ samples of tail vein blood collected in heparinized microcapillary tubes; the samples were centrifuged and glucose was determined in $10-\mu 1$ aliquots of plasma with a Beckman glucose analyzer (Beckman Instruments, Fullerton, Calif.). Plasma glucose concentration at sacrifice was determined in plasma prepared from heparinized blood obtained by cardiac puncture.

The details of the technique and instrumentation employed for the determination of sciatic MNCV have been published previously (21). In brief, on day 14 the rats were anesthetized with sodium pentobarbital $(0.04 \mathrm{mg} / \mathrm{g} \mathrm{wt}$, i.p. $)$, and the sciatic nerve was exposed surgically. The exposed tissues were warmed by radiant heat from a coil suspended $30 \mathrm{~cm}$ above the tissue, and controlled by a thermoregulator connected to a thermocouple taped to tissue neighboring the exposed nerves; the system provided a direct continuous read-out of tissue temperature. The nerve temperature was found to be similar to that of the surrounding tissues when checked with an electric thermometer at the end of each $\mathrm{MNCV}$ determination. In the experiments reported below the nerve temperatures were consistently maintained at 35 $\pm 1^{\circ} \mathrm{C}$.

The nerves were stimulated with $0.2 \mathrm{~ms}$ square-wave pulses at the sciatic notch and popliteal fossa through a pair of Teflon-coated electrodes with bare tips. At the completion of the experiment, the distance between the two points of stimulation was determined by measurement with a pair of calipers. The "different" electrode was applied to the nerve surface while the "indifferent" electrode was on neighboring tissue $2-4 \mathrm{~mm}$ away. The evoked action potentials of the triceps surae were led off by a concentric needle to channel 1 of the electromyograph. The pulses were derived from a double-screened stimulator driven by a timer, which also triggered the sweep of the electromyograph. A stimulus-marking unit and a current-metering unit wired to the stimulator permitted the display of a $50 \mu \mathrm{s}$ stimulus marker in channel 1, and the current in the other two channels of the electromyograph. The results were photographed by a movie camera with the interrupted mode of recording and a sweep speed of $0.25 \mathrm{~ms} / \mathrm{mm}$. The latencies (the time between the $50 \mu$ s stimulus-marker and the "takeoff" of the evoked action potential from the base line), peak-to-peak amplitudes of the evoked potential, motor nerve threshold, and supramaximal currents (8-12 times threshold current) were determined from the developed film. All measurements were made by calipers. MNCV was calculated by dividing the distance between the points of stimulation by the difference in the latencies from these two points. As previously reported, the mean MNCV determined in this fashion did not differ significantly over a period of $9 \mathrm{wk}$ in male Wistar rats, initial wt 120-150 g, whose body weight increased to an average of $325 \mathrm{~g}$ while maintained on a commercial rat chow (21).

After the determination of $\mathrm{MNCV}$, the contralateral sciatic nerve was exposed surgically and the segment of the nerve from the sciatic notch to the popliteal fossa was quickly removed and frozen in liquid nitrogen. The animals were then sacrificed by cardiac puncture, and the heparinized blood was centrifuged at $3,000 \mathrm{~g}$ for $15 \mathrm{~min}$ at $4^{\circ} \mathrm{C}$ and the plasma frozen rapidly. For the determination of plasma free MI, Somogyi filtrates were prepared from 1.0 $\mathrm{ml}$ of plasma, and an aliquot of the filtrate equivalent to $0.5 \mathrm{ml}$ of the original plasma was lyophilized after the addition of $50 \mu \mathrm{g}$ of $\alpha$-D-methyl mannopyranoside (Pfanstiehl Chemical Corp., Waukegan, Ill.), used as an internal standard. The hexa-O-trimethylsilyl ether of myoinositol was prepared by the method of Sweeley, Bentley, Makita, and Wells (22), with $1.0 \mathrm{ml}$ of pyridine-hexamethyldisilazanetrimethylchlorosilane $(10: 2: 1, \mathrm{vol} / \mathrm{vol} / \mathrm{vol})$; however, the reaction time was extended to $16 \mathrm{~h}$ with shaking. The trimethylchlorosilane was partially hydrolyzed and redistilled before use as described by Roberts $(23)$. Aliquots $(2-5 \mu 1)$ of the reaction mixture were injected onto a $180-\mathrm{cm}$ column of $3 \% \mathrm{JXR}$ on Gas-Chrom Q (Supelco, Inc., Bellefonte, $\mathrm{Pa}$.) and chromatographed at $180^{\circ} \mathrm{C}$ with $\mathrm{N}_{2}(50 \mathrm{ml} / \mathrm{min})$ as carrier in a Packard gas-liquid chromatographic apparatus equipped with a hydrogen flame detector (Packard Instrument Co., Inc., Downers Grove, Ill.). Standard curves were determined daily and the recovery of added free MI consistently exceeded $90 \%$. To determine nerve free MI concentration, the frozen nerves were cleaned of any adherent blood, weighed, transferred to a glass homogenizing 
TABLE I

Effects of Streptozotocin Diabetes and Insulin Treatment on Sciatic MNCV

\begin{tabular}{lccccc}
\hline \multicolumn{1}{c}{ Type of rat } & Diet & $n$ & Weight* & Plasma glucose & MNCV§ \\
\hline & & & $g$ & $m g / d l$ & $M / s$ \\
Normal & Standard & 20 & $247 \pm 6$ & $167 \pm 9$ & $64.6 \pm 0.9$ \\
Diabetic & Standard & 35 & $178 \pm 9$ & $539 \pm 23$ & $50.1 \pm 0.9$ \\
Insulin-Rx diabetics & & & & & \\
$\quad$ Group 1 & Standard & 9 & $235 \pm 6$ & $214 \pm 40$ & $51.4 \pm 1.1$ \\
Group 2 & Standard & 14 & $254 \pm 6$ & $213 \pm 18$ & $51.1 \pm 1.7$ \\
$\quad$ Group 3 & Standard & 17 & $280 \pm 5$ & $75 \pm 18$ & $62.9 \pm 0.9$ \\
Normal & & & & & \\
Diabetic & Chow & 14 & $239 \pm 3$ & $168 \pm 4$ & $65.0 \pm 1.6$ \\
Streptozotocin controls & Chow & 16 & $175 \pm 6$ & $528 \pm 25$ & $47.6 \pm 1.6$ \\
& Chow & 9 & $209 \pm 7$ & $168 \pm 4$ & $65.5 \pm 1.1$ \\
\hline
\end{tabular}

Results are means \pm SEM.

* The weights listed are the mean for each group on day 14 .

$\ddagger$ The plasma glucose values represent the mean for each group in samples obtained by cardiac puncture at the time of sacrifice. The exceptions are the three groups of insulin-treated diabetics, in which the values listed are the mean of the average tail vein plasma glucose concentration observed in each animal in the group during the last week of the study.

$\S$ The MNCV is the mean value observed in each group on day 14 .

vessel containing $2.6 \mathrm{ml}$ of boiling water, and kept in boiling water for $3 \mathrm{~min} ; 0.2 \mathrm{ml}$ of $5 \% \mathrm{ZnSO}_{4}$ was added and the nerve was homogenized by hand with a glass pestle, 0.2 $\mathrm{ml}$ of $0.3 \mathrm{~N} \mathrm{Ba}(\mathrm{OH})_{2}$ was added, and the homogenization was repeated. The homogenate was centrifuged and an aliquot of a supernate $(2.0 \mathrm{ml})$ was used for the analysis of MI as described for plasma. For the determination of sciatic nerve free glucose, sorbitol, and fructose, the nerve was removed and quickly frozen in liquid $\mathrm{N}_{2}$, as described above. The frozen nerve was cleaned of any adherent blood, weighed, and homogenized in $1.0 \mathrm{ml} 6 \% \mathrm{HClO}_{4}$ at $4^{\circ} \mathrm{C}_{\text {. }}$ Neutralized perchloric acid filtrates were then prepared and aliquots analyzed for sorbitol and fructose by the fluorometric enzymatic assays, previously described (24), and for free glucose by a spectrophotometric enzymatic assay (25).

To determine the free MI content of the chow diet, triplicate $5.0-\mathrm{g}$ aliquots of the ground chow were ground to a fine powder in a mortar, and then extracted three times with $100 \mathrm{ml}$ of boiling water with stirring for $30 \mathrm{~min}$. The combined extracts were filtered, flash-evaporated at $80^{\circ} \mathrm{C}$, and reconstituted in $10 \mathrm{ml}$ of $\mathrm{H}_{2} 0 ; 3.0 \mathrm{ml}$ of $5 \% \quad \mathrm{ZnSO}_{4}$ and $3.0 \mathrm{ml}$ of $0.3 \mathrm{~N} \mathrm{Ba}(\mathrm{OH})_{2}$ were then added and the supernate was recovered after centrifugation. Aliquots of the extract were then analyzed for free MI after the addition of the internal standard, as described for plasma.

\section{RESULTS}

The induction of streptozotocin diabetes consistently resulted in impaired sciatic MNCV within 14 days, both in rats fed the standard diet, which contained all known vitamin requirements and $0.011 \% \mathrm{MI}$, and in those fed a commercial show (Table I). Impaired MNCV did not develop in rats that failed to become hyperglycemic after streptozotocin administration (Table I). Our initial efforts to prevent the development of impaired MNCV by insulin treatment, started 3 days after streptozotocin administration, were unsuccessful (Table I, groups 1 and 2 ). In these diabetic rats insulin treatment resulted in improved or normal weight gain, and in a decrease in their degree of hyperglycemia, but the mean MNCV on day 14 in both groups was not significantly different from that of the untreated diabetics. A wider range of MNCV was observed in group 2, as indicated by the larger SEM (Table I), and a few rats in this group had MNCV's that approached the normal range. Although these observations were similar to those of Eliasson (12), it was apparent that the insulin-treated diabetic rats in groups 1 and 2 frequently had elevated plasma glucose concentrations during the period of treatment; during the last week of the study the mean $\pm S E M$ of the average plasma glucose concentration (tail vein) observed in each animal in group 1 was $214 \pm 40 \mathrm{mg} / \mathrm{dl}$, and the comparable value for group 2 was $213 \pm 18 \mathrm{mg} /$ dl. The plasma glucose concentration (tail vein) in a group of normal rats fed the same standard diet averaged $152 \pm 4 \mathrm{mg} / \mathrm{dl}(n=11)$ on the morning of day 14 ; serial determinations of plasma glucose were not performed in the normal rats whose data are given in Table I.

A group of 24 diabetic rats was subjected to insulin treatment designed to prevent any prolonged period in which their plasma glucose concentration exceeded that observed in the normal rats fed the same diet, with $152 \pm 4 \mathrm{mg} / \mathrm{dl}$ as an admittedly somewhat arbitrary estimate of the upper range of plasma glucose fluctuation in the normals. As detailed in Methods, the diabetic rats in group 3 received twice-daily injections of isophane insulin, and the insulin dosage was adjusted according to 
the plasma glucose concentration, which was determined twice daily on most days. The pattern of plasma glucose fluctuation observed in the insulin-treated diabetics in group 3 is illustrated in Fig. 1; the mean plasma glucose concentration (tail vein) in this group was never found to exceed $160 \mathrm{mg} / \mathrm{dl}$ during the week before the determination of $\mathrm{MNCV}$ on day 14. As noted in Methods, hypoglycemia was a problem in the animals in group 3, and 7 of the 24 rats succumbed before day 14 ; the mean $\pm S E M$ of the average plasma glucose concentration in each of the 17 survivors during the last week of the study was $75 \pm 18 \mathrm{mg} / \mathrm{dl}$. The average weight of the rats in group 3 on day 14 was $19 \%$ higher than that of the insulin-treated diabetics in group 1, $10 \%$ higher than that of group 2, and $13 \%$ higher than that of the normal controls (Table I). As shown in Table I, the mean sciatic $\mathrm{MNCV}$ in the insulin-treated diabetics of group 3 on day 14 was not significantly different from that of the normal rats.

The concentration of free MI in the sciatic nerves of normal rats fed the standard diet was 90 -fold higher than that found in their plasma (Table II). There was no significant difference between the plasma free MI concentrations in normal and diabetic rats fed the standard diet (Table II). However, the concentration of free MI in the sciatic nerves of the diabetics was significantly lower after $2 \mathrm{wk}$, when compared with nerves from normal rats fed the same standard diet (Table II). Insulin treatment adequate to prevent the development of impaired sciatic MNCV in the diabetic rats in group 3 appeared to prevent any significant decrease in nerve free MI concentration (Table II). In the insulin-treated rats in group 1 , the sciatic nerve free MI content after
2 wk was similar to that found in the untreated diabetics (Table II). In the insulin-treated diabetic rats in group 2 , which had a wider range of $\mathrm{MNCV}$ on day 14 , there was a significantly higher nerve free MI content than in the untreated diabetics (Table II). In group 2 there was a positive linear correlation between the sciatic nerve free MI content on day 14 and MNCV $(r=+$ $0.72, P<0.001)$. This correlation was not apparent in any other group of normal, diabetic, or insulin-treated diabetic rats. These observations suggested that there might be some relationship between the development of impaired $\mathrm{MNCV}$ in rats with acute experimental diabetes and the associated decrease in nerve free MI content.

Although active concentration of free MI has been demonstrated in lens $(26,27)$, kidney cortex slices (28), and intestinal mucosa (29), Stewart, Kurien, Sherman, and Cotlier (30) have suggested that in most mammalian tissues, including sciatic nerve, the tissue free MI concentrations reflect a steady state between endogenous synthesis, slow leakage from cells, and possible catabolism through glucuronic acid. (The last process appears to be essentially restricted to the kidney in the rat [31]). These workers found no difference in the plasma or sciatic nerve free MI concentrations in normal female rats fed a MI-deficient diet or the same diet supplemented with $0.0125 \% \mathrm{MI}$ (30). Consequently, it has been assumed that the free MI content of peripheral nerves is largely independent of dietary intake. However, in the course of these studies we found that normal male rats fed the chow diet maintained significantly higher plasma and sciatic nerve free MI concentrations than rats fed the standard diet (Table II). On analysis,

TABLE II

Effects of Streptozotocin Diabetes, Insulin Treatment, and Dietary Free MI Content on the Concentration of Free MI in Plasma and Peripheral Nerve on Day 14

\begin{tabular}{llcccc}
\hline \multicolumn{1}{c}{ Type of rat } & \multicolumn{1}{c}{ Diet } & $n$ & Plasma free MI & Nerve free MI & $P$ \\
\hline & & & $m M$ & $m m o l / k g$ wet wt & \\
Normal & Standard & 10 & $0.034 \pm 0.003$ & $3.17 \pm 0.25$ & $<0.005$ \\
Diabetic & Standard & 15 & $0.034 \pm 0.004$ & $2.19 \pm 0.11$ \\
Insulin-Rx diabetics & & & & \\
$\quad$ Group 1 & Standard & 9 & $0.039 \pm 0.003$ & $2.52 \pm 0.13$ \\
$\quad$ Group 2 & Standard & 14 & $0.035 \pm 0.003$ & $2.82 \pm 0.19$ & $<0.005$ \\
$\quad$ Group 3 & Standard & 17 & $0.030 \pm 0.002$ & $2.98 \pm 0.22$
\end{tabular}

Values listed are the mean \pm SEM for each group. The $P$ values were determined by Student's $t$ test. 
TABLE III

Effects of Dietary MI Content on MNCV in Normal and Diabetic Rats

\begin{tabular}{llccccc}
\hline Type of rat & \multicolumn{1}{c}{ Diet } & $n$ & $\mathrm{Wt}$ & Plasma glucose & MNCV & $P$ \\
\hline & & & $g$ & $m g / d l$ & $M / s$ \\
Normal & Standard & 20 & $247 \pm 6$ & $167 \pm 9$ & $64.6 \pm 0.9$ & \\
Diabetic & Standard & 35 & $178 \pm 9$ & $539 \pm 23$ & $50.1 \pm 0.9$ & \\
Normal & Standard +1\% MI & 14 & $239 \pm 5$ & $188 \pm 8$ & $64.2 \pm 1.4$ & $<0.001$ \\
Diabetic & Standard +1\% MI & 19 & $164 \pm 8$ & $552 \pm 9$ & $58.0 \pm 1.4$ & \\
Normal & Chow & 14 & $239 \pm 3$ & $168 \pm 4$ & $65.0 \pm 1.6$ & $<0.001$ \\
Diabetic & Chow & 16 & $175 \pm 6$ & $528 \pm 25$ & $47.6 \pm 1.6$ & \\
Normal & Chow $+1 \%$ MI & 5 & $219 \pm 12$ & $158 \pm 7$ & $63.8 \pm 1.4$ & NS \\
Diabetic & Chow +1\% MI & 18 & $169 \pm 6$ & $572 \pm 25$ & $60.5 \pm 0.9$ & \\
\hline
\end{tabular}

All values are means $\pm \mathrm{SEM}$ for determinationsc arried out on day 14. Plasma glucose was determined in samples obtained by cardiac puncture at sacrifice.

the commercial chow was found to contain approximately $0.069 \%$ free MI by weight (see Methods). These observations suggested that the concentration of free $\mathrm{MI}$ in normal rat sciatic nerve might be subject to modification by dietary MI content and plasma free MI concentration. To test the latter possibility, two groups of normal rats (10 each) that had been maintained for several days on the chow diet were fasted overnight, and then given an i.p. injection of either $0.20 \mathrm{ml}$ saline or $0.20 \mathrm{ml}$ saline containing $10 \mathrm{mg}$ free $\mathrm{MI} / 100 \mathrm{~g}$ body wt. (In preliminary experiments the peak in plasma free MI after a similar MI load was found to occur at $1-2 \mathrm{~h}) .4 \mathrm{~h}$ after the injection, the plasma free MI averaged $0.087 \pm 0.010 \mathrm{mM}$ in rats given MI i.p., as compared with $0.050 \pm 0.003 \mathrm{mM}$ in the fasted saline-injected controls. The sciatic nerve free MI content $4 \mathrm{~h}$ after the injection of the MI load was $4.18 \pm 0.33 \mathrm{mmol} / \mathrm{kg}$ as compared with $3.38 \pm 0.18$ in the controls, $P<0.05$. It would appear that relatively rapid increases in sciatic nerve free MI content can be induced in normal rats by raising the plasma free MI content. It seems reasonable to speculate that this may reflect the effect of plasma MI concentration on the active concentration of free MI by components of the peripheral nerve, or on its release by the tissue.

In diabetic rats fed the chow diet, the decreased sciatic MNCV observed after 2 wk was also associated with a lower sciatic nerve free MI content than that observed in normal rats fed the same diet (Table II). It will be noted, however, that the absolute nerve free MI concentrations in the normal and diabetic rats fed the chow diet were higher than those observed in nerves from comparable groups fed the standard diet (Table II).

The effects of supplementing the standard diet with pharmacological quantities of free MI were examined. Normal and diabetic rats were maintained on the standard diet supplemented with $1 \%$ free MI for 2 wk. As shown in Table II, this resulted in a fivefold increase in plasma free MI in both normals and diabetics, the plasma concentrations in both groups being approximately $0.20 \mathrm{mM}$. Under these conditions, there was an increase in the free MI content of the sciatic nerves in the normals, and the difference between the nerve free MI content in the diabetics and normals on day 14 was essentially abolished (Table II). As shown in Table III, supplementing the standard diet with $1 \%$ free MI had no effect on MNCV in the normal rats; however, in the diabetic rats it resulted in a marked improvement in the MNCV, observed after $2 \mathrm{wk}$, when compared with diabetics maintained on the standard diet. It should be noted that both groups of diabetic rats exhibited similar degrees of severe persistent hyperglycemia (Table III). These studies were repeated in normal and diabetic rats fed the chow diet supplemented with $1.0 \%$ MI. After 2 wk there was no difference in the sciatic nerve free MI content of the diabetic and normals, and the development of impaired MNCV in the diabetics was virtually prevented despite severe persistent hyperglycemia (Tables II and III).

If impaired sciatic nerve $\mathrm{MNCV}$ was permitted to develop in diabetics fed the standard diet for $2 \mathrm{wk}$, and the diet was then supplemented with $1 \%$ free MI, there was a marked improvement in MNCV over the ensuing $2 \mathrm{wk}$ (Fig. 2). However, the defect in MNCV persisted in the diabetic rats that continued to eat the standard diet (Fig. 2).

As anticipated, the beneficial effects of dietary myoinositol supplementation on the development of impaired $\mathrm{MNCV}$ in diabetic rats were restricted to diets that did not elevate the plasma free MI concentration beyond a critical range. This expectation was derived from previous studies in which we examined the possible relationship between the markedly elevated plasma free MI concentrations found in patients with chronic uremia and the development of the impaired MNCV as- 


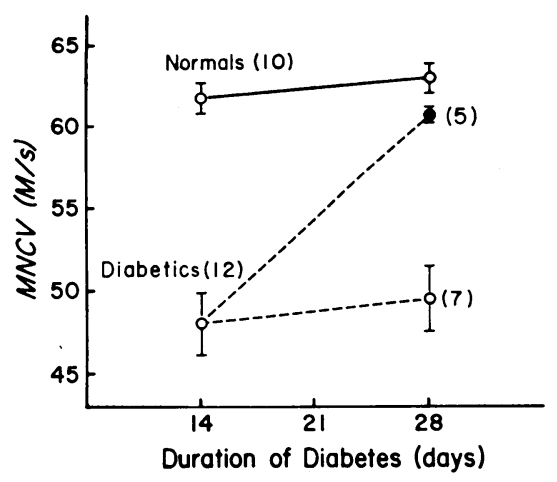

FIGURE 2 Sequential determinations of sciatic MNCV in streptozotocin-diabetic rats on days 14 and 28 after streptozotocin administration. The diabetics were all maintained on the standard diet until day 14; five diabetics were then transferred to the same diet supplemented with $1.0 \%$ free MI ( ), and seven maintained on the standard diet (O) ; MNCV was determined in the contralateral sciatic nerve on day 28. Sequential determinations of $\mathrm{MNCV}$ in normal rats fed the standard diet throughout the same period are also depicted. Values represent means \pm SEM for the groups.

sociated with that syndrome $(21,32)$. In those studies we observed that the plasma free MI in normal rats can be chronically elevated to levels similar to those observed in chronic human uremics (approximately 0.50 $\mathrm{mM}$ ) by feeding diets containing $35 \%$ free MI or by the repeated i.p. injections of large free MI loads. These rats developed decreased sciatic $\mathrm{MNCV}$ within $1 \mathrm{wk}$, which improved over a period of $1 \mathrm{wk}$ if the plasma free MI was permitted to fall to normal levels. Fig. 3 illustrates the effects of feeding normal and diabetic rats the standard diet supplemented with $3 \%$ free MI. On this diet the normal rats had plasma free MI concentrations that averaged $0.349 \pm 0.016 \mathrm{mM}(n=11)$, and this was

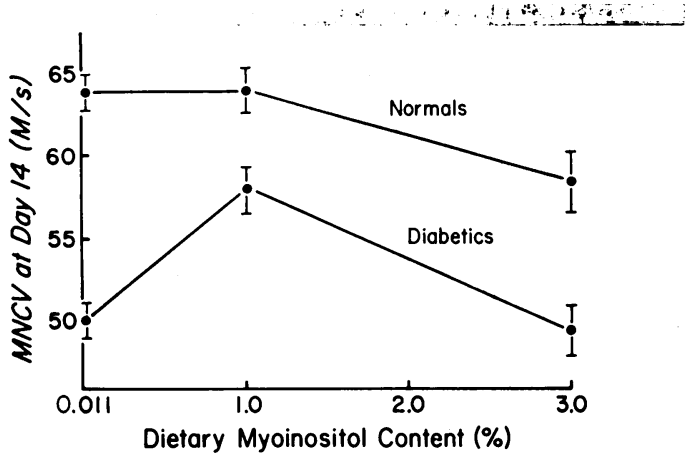

FIGURE 3 Effects of varying dietary free MI content on the MNCV observed after 2 wk in groups of normal rats and in diabetic rats that had received streptozotocin 14 days previously. The diets were the standard diet (free MI content $0.011 \%$ ) or pellets prepared from the standard diet supplemented with $1.0 \%$ or $3.0 \%$ free MI by weight. Values represent means $\pm \mathrm{SEM}$ for each group. $n$ 's for normals are $(0.011 \%) 20,(1.0 \%) 14,(3.0 \%) 11$. n's for the diabetics are $(0.011 \%) 35,(1.0 \%) 19,(3.0 \%) 13$. associated with a small but significant decrease in MNCV after 2 wk. On the diet supplemented with $3 \%$ free MI, the diabetic rats had plasma MI levels that averaged $0.499 \pm 0.046 \mathrm{mM}(n=13)$, and these animals exhibited a significantly lower mean sciatic MNCV after 2 wk than diabetics fed the standard diet supplemented with $1 \%$ free MI (Fig. 3 ).

There has been speculation that the development of impaired sciatic MNCV in diabetic rats may be related to increased polyol pathway activity in components of the peripheral nerve that is induced by a rise in intracellular glucose concentration (33). It is known that experimental diabetes results in increased concentrations of glucose, sorbitol, and fructose in the sciatic nerves of rats with experimental diabetes, and that these concentrations fall rapidly when the plasma glucose is lowered by insulin administration (34). To exclude the possibility that the elevated plasma free MI concentrations, resulting from supplementing the normal diets with $1.0 \%$ free MI, might affect the development of impaired $\mathrm{MNCV}$ in diabetic rats by altering nerve glucose concentrations or polyol pathway activity, glucose, sorbitol, and fructose were determined in the sciatic nerves of diabetic rats fed the standard diet or the standard diet $+1.0 \%$ free MI for $2 \mathrm{wk}$; no significant differences were observed. In the diabetics fed the standard diet, plasma glucose was $26.6 \pm 3.6 \mathrm{mM}$ on day 14 , and sciatic nerve glucose was $13.0 \pm 1.2 \mathrm{mmol} / \mathrm{kg}$, sorbitol $2.69 \pm 0.21 \mathrm{mmol} / \mathrm{kg}$, and fructose $7.38 \pm 0.53$ $\mathrm{mmol} / \mathrm{kg}, n=5$. In the diabetics fed the standard diet $+1.0 \%$ free MI, plasma glucose was $29.1 \pm 2.2 \mathrm{mM}$, nerve glucose was $14.7 \pm 1.0$, nerve sorbitol $2.68 \pm 0.41$, and nerve fructose $7.18 \pm 0.57 \mathrm{mmol} / \mathrm{kg}, n=5$. It thus appears unlikely that the effect of increased dietary free MI content on the development of impaired MNCV in diabetic rats results from inhibition of polyol pathway activity in peripheral nerve.

\section{DISCUSSION}

The development of impaired sciatic $\mathrm{MNCV}$ in rats with acute experimental diabetes appears to be independent of the specific means employed for its induction (i.e., pancreatectomy [12], alloxan (12), or streptozotocin). The experiments reported above demonstrate that the development of impaired $\mathrm{MNCV}$ can be prevented by insulin treatment. The data are compatible with the conclusion that to be effective in preventing impaired $\mathrm{MNCV}$, insulin therapy must prevent any prolonged period of hyperglycemia. However, the insulin-treated diabetics in whom rigorous efforts were made to prevent prolonged periods of hyperglycemia (group 3) could be considered relatively hypoglycemic during the last week of the study, and we cannot exclude the possibility that this contributed to the preven- 
tion of impaired MNCV. Although the rats in group 3 also gained more weight than the diabetic rats in which insulin treatment failed to prevent a decrease in $\mathrm{MNCV}$ (groups 1 and 2), the mean weights in the latter groups were not significantly different from that of the nor-

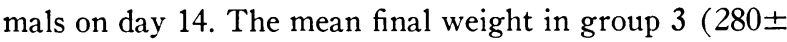
$5 \mathrm{~g}$ ) was $13 \%$ greater than that of the normals, but we have previously reported that increases in body weight in normal male Wistar rats from $120-150 \mathrm{~g}$ to $325 \mathrm{~g}$ are not associated with significant changes in sciatic MNCV under the conditions employed in this study (21). It seems reasonable to conclude that metabolic derangements resulting from insulin deficiency play a primary role in the pathogenesis of the impaired peripheral MNCV found in acute experimental diabetes in rats. This relationship has been obscured for some time by the fact that insulin treatment that results in normal weight gain and a decrease in the degree of hyperglycemia appears inadequate consistently to prevent the development of impaired peripheral nervous function.

The development of impaired peripheral nerve conduction in acute experimental diabetes seems to occur in the absence of demonstrable morphological alterations. In a recent study Sharma and Thomas found no evidence of a decrease in myelinated nerve fiber diameter, of segmental demyelination, of paranodal demyelination, or of axonal breakdown in the sural nerves of rats with streptozotocin diabetes of 3 and $6 \mathrm{wk}$ duration (15). However, the electrical properties of the peripheral nerve have been found to be altered by acute experimental diabetes. Eliasson found that alloxan diabetes of 10 days duration results in a marked decrease in the electrical resistance of internodal segments of large myelinated fibers dissected from rat tibial nerve, without any change in internodal capacitance (35). Within the limits of the methods employed, the electrical characteristics of the nodes of Ranvier were unaltered. Eliasson suggested that the development of "leaky" myelin in the internodes of large myelinated fibers might contribute to the decrease in nerve conduction velocity, and noted that this could result from myelin edema or from a change in myelin composition (35).

The studies reported above suggest that acute experimental diabetes results in an alteration in the metabolism of free MI in peripheral nerve, which has some relationship to the development of impaired peripheral MNCV. The components of the peripheral nerve normally maintain a very high tissue-to-plasma free MI concentration gradient. The absolute free MI concentration within the peripheral nerve appears to vary with anatomical location, and there is indirect evidence that a large fraction of the free MI may be localized within Schwann cells (36). It has been assumed that the major source of the free MI found in peripheral nerve is derived from synthesis in situ (30), and quantification of free MI synthesis may prove the correctness of this assumption. However, the free MI content of peripheral nerve can be acutely increased by the i.p. injection of a free MI load, and is increased by increasing dietary free MI content associated with increases in plasma free MI concentration. This suggests that active concentration of free MI may occur in some components of the peripheral nerve, or alternatively, that free MI is released from peripheral nerve at a rapid rate that can be modified by increases in plasma free MI concentration.

In rats with acute streptozotocin diabetes the development of impaired MNCV over a period of $2 \mathrm{wk}$ is associated with a decrease in the free MI content of the nerve, as compared with nerves from normal rats fed the same diet, provided that the dietary free MI content is within a presumably normal range. This decrease in nerve free MI content occurs despite plasma free MI concentrations similar to those found in normals fed the same diet. Insulin treatment adequate to prevent impaired $\mathrm{MNCV}$ also prevents any significant alteration in nerve free MI content. The bulk of our data provides no evidence for a direct correlation between the absolute nerve free MI concentration and MNCV; however, such a relationship was apparent in one group of inadequately insulin-treated diabetics. The studies reported above indicate that the development of $\mathrm{MNCV}$ in rats with acute experimental diabetes can be modified or totally prevented by supplementing the diet with $1 \%$ free $\mathrm{MI}$; on such diets the diabetics have moderately elevated plasma free MI concentrations and they maintain sciatic nerve free MI concentrations that are not significantly different from the elevated levels found in the nerves of normal rats fed the same diet. It seems reasonable to speculate that elevations in plasma free MI within a critical range may compensate for impaired active concentration of free MI in some components of peripheral nerve in the diabetic, and it appears that under these conditions the development of impaired MNCV is ameliorated or prevented without insulin treatment and despite severe persistent hyperglycemia.

The factors responsible for the decreased nerve free MI concentrations in diabetic rats maintained on diets with a normal free MI content cannot be discerned from the data available, and the extent to which the diverse cellular components of the peripheral nerve share in this alteration remains to be clarified. Alloxan diabetes is known to result in a decrease in the free MI content of the lens (37). There is evidence for the active concentration of free MI in that tissue $(26,27)$, and in lenses incubated with elevated concentrations of glucose or galactose, the uptake of labeled MI is decreased and there is an increase in MI efflux $(26,37)$. The basis of these alterations has not been firmly established, but 
both glucose and galactose are substrates for lens aldose reductase, and it has been suggested that the derangement in the regulation of lens free MI concentration may be a secondary consequence of the osmotic effects of the associated increases in lens sorbitol or galactitol (30, 38). Experimental diabetes results in increased concentrations of free glucose, sorbitol, and fructose in rat sciatic nerve, and these fall rapidly when the plasma glucose is lowered by insulin administration (13, 34). In rats with chronic experimental galactosemia induced by galactose feeding, there is a marked decrease in sciatic nerve free MI content in association with high concentrations of galactitol (34). Thus there may be some relationship between increased polyol pathway activity in the peripheral nerves of rats with acute experimental diabetes and the impaired regulation of nerve free MI concentration. However, the basis for such a relationship is still obscure, since there is no evidence, as yet, that hyperglycemia results in osmotic swelling of components of the peripheral nerve (13).

The physiological significance of the high free MI concentrations found in peripheral nerve and other mammalian tissues is unknown, and so there is no firm basis for speculation concerning the possible effects of alterations in nerve free MI content. The known metabolic fates of myoinositol in neural tissue include its incorporation into phosphatidyl inositol (39), the parent compound of the polyphosphoinositides (40). The latter are present in significant concentrations in peripheral nerve, and may be largely associated with myelin (41). There has been continuing interest and controversy concerning the role that the turnover of specific inositolcontaining phospholipids may play in cation transport and neural transmission $(40,41)$. The possibility that the decreased nerve free MI concentrations found in acute experimental diabetes may be associated with alterations in the metabolism of the MI-containing phospholipids would therefore merit examination.

The experiments in this report pertain solely to alterations in peripheral nerve associated with acute experimental diabetes, and their significance with regard to the pathogenesis of diabetic polyneuropathy remains to be assessed. It must be noted that Sharma and Thomas (15) have recently reported studies of the function and morphology of rats with chronic streptozotocin and alloxan diabetes and were unable to demonstrate any significant morphological alterations in their peripheral nerves. These workers concluded that "the extrapolation of biochemical findings in experimental diabetes in an attempt to explain the origin of human diabetic neuropathy, where there are associated structural changes should be made with caution." We agree with this conclusion, but feel that it may be premature to dismiss the changes in function and metabolism found in acute experimental diabetes or to abandon efforts to develop an appropriate animal model for overt polyneuropathy.

There has been no systematic study in experimental animals of the possibility that the alterations induced in peripheral nerve by acute insulin deficiency may modify the effects of independent factors (e.g. local pressure, nutritional deficiencies) that could be operative in the diabetic population. In addition, none of the studies of the effects of chronic experimental diabetes on peripheral nervous function or morphology have been carried out under conditions in which factors that may affect plasma and nerve free MI concentrations have been controlled. Dietary free MI content is rarely determined, and the effects of chronic experimental diabetes on free MI absorption require reexamination. It is of note that Stewart, Sherman, Kurien, Moonsammy, and Wisgerhof (34) found only small and statistically insignificant decreases in the free MI content of sciatic nerves in female alloxan-diabetic rats after 4 mo of disease (MNCV was not examined). However, Stewart et al. (34) found that the plasma MI in these diabetics averaged $0.09 \pm$ $0.01 \mathrm{mM}$, and levels of $0.08 \pm 0.01 \mathrm{mM}$ were present in the controls. These high plasma MI levels in the controls suggest that the diet employed in these studies may have contained significantly higher concentrations of free MI than our standard or chow diets. The dietary free MI content was not specified (34), but the same authors reported plasma levels of $0.03 \mathrm{mM}$ in normal male rats fed a diet with a free MI content similar to that of our standard diet (30).

In addition to controlling dietary free MI content, the effects of renal disease will have to be considered in evaluating the effects of chronic experimental diabetes on the morphology and function of peripheral nerve. The studies reported above suggest that alterations in plasma free MI resulting from renal disease may have beneficial or adverse effects on the derangements responsible for impaired $\mathrm{MNCV}$, depending upon the resulting range of plasma free MI concentration. Alloxan has the potential of inducing acute renal damage (42), and alterations in renal morphology have been reported in rats with chronic streptozotocin diabetes $(43,44)$. In a prior study, we examined $\mathrm{MNCV}$ in rats with chronic streptozotocin diabetes of 14 mo duration maintained at the Merck Research Institute by Drs. M. Glitzer and H. Katzen (45). The mean MNCV in these diabetics with persistent hyperglycemia was not significantly different from that of their age- and sex-matched controls, and there was no difference in the mean sciatic nerve free MI content in the two groups. However, the diabetic rats had a mean plasma urea nitrogen concentration (42 $\mathrm{mg} / \mathrm{dl}$ ) significantly higher than that of the controls $(17 \mathrm{mg} / \mathrm{dl})$, and the diabetics had significantly higher plasma free MI con- 
centrations, which were linearly related to their plasma urea nitrogen concentrations. In the normal rats there was no relationship between plasma free MI and sciatic $\mathrm{MNCV}$, but in the diabetics there was a highly significant linear relationship between increasing plasma free MI concentration and increasing MNCV (45).

Finally, it must be noted that the manner in which axonal flow may be affected by insulin deficiency or other factors operative in rats with experimental diabetes remains to be examined; this information may be critical to the development of an appropriate model for diabetic polyneuropathy if, as suggested, the development of pathological lesions in human diabetics is, in part, a reflection of a "dying back" phenomenon (46). For these reasons, it would appear that the relationship of insulin deficiency to the development of pathological lesions in peripheral nerve is still an open question.

\section{ACKNOWLEDGMENTS}

The authors thank Mrs. Sarah Ann Aquino and Mrs. Joan Feuer for expert assistance.

These studies were supported in part by U. S. Public Health Service Grants AM-04722 and AM-05556, and a gift from the Ware Fcundation.

\section{REFERENCES}

1. Thomas, P. K., and R. G. Lascelles. 1966. The pathology of diabetic neuropathy. Q. J. Med. New Series 35: 489-509.

2. Greenbaum, D., P. C. Richardson, M. V. Salmon, and H. Urich. 1964. Pathological observations on six cases of diabetic neuropathy. Brain. 87: 201-214 and plates XXXIX-X.LIII.

3. Chopra, J. S., L. J. Hurwitz, and D. A. D. Montgomery. 1969. The pathogenesis of sural nerve changes in diabetes mellitus. Brain. 92: 391-418.

4. Chopra, J. S., and L. J. Hurwitz. 1969. A comparative study of peripheral nerve conduction in diabetes and non-diabetic chronic occlusive peripheral vascular disease. Brain. 92: 83-96.

5. Bischoff, A. 1973. Ultrastructural pathology of peripheral nervous system in early diabetes. Adv. Metab. Disord. Suppl. 2 : 441-449.

6. Locke, S. 1971. The nervous system and diabetes. In Joslin's Diabetes Mellitus. A. Marble, P. White, R. F. Bradley, and L. P. Krall, editors. 11th edition. Lea \& Febiger, Philadelphia. Pa. 562-580.

7. Gilliatt, R. W., and R. G. Willison. 1962. Peripheral nerve conduction in diabetic neuropathy. J. Neurol. Neurosurg. Psychiatry. 25: 11-18.

8. Noël, P., J. P. Lauvaux, and J. Pirart. 1971. Upper limbs diabetic neuropathy: a clinical and electrophysiological study. Horm. Metab. Res. 3: 386-392.

9. Lawrence, D. G., and S. Locke. 1961. Motor nerve conduction velocity in diabetes. Arch. Neurol. 5: 483489.

10. Gregersen, G. 1968. Variations in motor conduction velocity produced by acute changes of the metabolic state in diabetic patients. Diabctologia. 4: 273-277.

11. Gregersen, G. 1967. Diabetic neuropathy: influence of age, sex, metabolic control, and duration of diabetes on motor conduction velocity. Neurology. 17: 972-980.
12. Eliasson, S. G. 1964. Nerve conduction changes in experimental diabetes. J. Clin. Invest. 43: 2353-2358.

13. Gabbay, K. H. 1973. Role of sorbitol pathway in neuropathy. Adv. Mctab. Disord. Suppl. 2: 417-424.

14. Preston, G. M. 1967. Peripheral neuropathy in the alloxan-diabetic rat. J. Physiol. (Lond.). 189: 49P-50P.

15. Sharma, A. K., and P. K. Thomas. 1974. Peripheral nerve structure and function in experimental diabetes. J. Ncurol. Sci. 23: 1-15.

16. Dawson, R. M. C., and N. Freinkel. 1961. The distribution of free mesoinositol in mammalian tissues, including some observations on the lactating rat. Biochem. J. 78: 606-610.

17. Sherman, W. R., M. A. Stewart, M. M. Kurien, and S. L. Goodwin. 1968. The measurement of myo-inositol, $m y o$-inosose-2 and scyllo-inositol in mammalian tissues. Biochim. Biophys. Acta. 158: 197-205.

18. Daughaday, W. H., and J. Larner. 1954. The renal excretion of inositol in normal and diabetic human beings. J. Clin. Invest. 33: 326-332.

19. Cunha, T. J. 1971. Inositol requirements of animals. In The Vitamins. W. H. Sebrell, Jr., and R. S. Harris, editors. Academic Press, Inc., New York. 3: 410-412.

20. Milhorat, A. T. 1971. Inositols: requirements of human beings. In The Vitamins, W. H. Sebrell, Jr., and R. S. Harris, editors. Academic Press, Inc., New York. 3: 412-415.

21. De Jesus, P. V., Jr., R. S. Clements, Jr., and A. I. Winegrad. 1974. Hypermyoinositolemic polyneuropathy in rats. A possible mechanism for uremic polyneurcpathy. J. Ncurol. Sci. $21: 237-249$.

22. Sweeley, C. C., R. Bentley, M. Makita, and W. W. Wells. 1963. Gas-liquid chromatography of trimethylsilyl derivatives of sugars and related substances. $J$. Am. Chem. Soc. 85: 2497-2507.

23. Roberts, R. N. 1967. Gas chromatography of inositol and glycerol. Lipid Chromatogr. Anal. 1: 447-463.

24. Travis, S. F., A. D. Morrison, R. S. Clements, Jr., A.I. Winegrad, and F. A. Oski. 1971. Metabolic alterations in the human erythrocyte produced by increases in glucose concentration. The role of the polyol pathway. J. Clin. Invest. 50: 2104-2112.

25. Slein, M. W. 1963. D-Glucose determination with hexokinase and glucose-6-phosphate dehydrogenase. In Methods of Enzymatic Analysis. H. U. Bergmeyer, editor. Academic Press, Inc., New York. 117-123.

26. Varma, S. D., B. Chakrapani, and V. N. Reddy. 1970. Intraocular transport of myoinositol. II. Accumulation in the rabbit lens in vitro. Invest. Ophthalmol. 9: 794800.

27. Cotlier, E. 1970. Myo-inositol: active transport by the crystalline lens. Invest. Ophthalmol. 9: 681-691.

28. Hauser, G. 1965. Energy and sodium-dependent uptake of inositol by kidney cortex slices. Biochem. Biophys. Res. Commun. 19: 696-701.

29. Caspary, W. F., and R. K. Crane. 1970. Active transport of $m y{ }^{\prime}$-inositol and its relation to the sugar transport system in hamster small intestine. Biochim. Biophy's. Acta. 203: 308-316.

30. Stewart, M. A., M. M. Kurien, W. R. Sherman, and E. V. Cotlier. 1968. Inositol changes in nerve and lens of galactose fed rats. J. Ncurochcm. 15: 941-946.

31. Howard, C. F., Jr., and L. Anderson. 1967. Metabolism of $m y$ y-inositol in animals. II. Complete catabolism of $m y$ 'o-inositol ${ }^{14} \mathrm{C}$ by rat kidney slices. Arch. Biochem. Biophys. 118 : 332-339. 
32. Clements, R. S., Jr., P. V. De Jesus, Jr., and A. I. Winegrad. 1973. Raised plasma-myoinositol levels in uraemia and experimental neuropathy. Lancet. 2: 11371141.

33. Gabbay, K. H. 1973. The sorbitol pathway and the complications of diabetes. N. Engl. J. Med. 288: 831-836.

34. Stewart, M. A., W. R. Sherman, M. M. Kurien, G. I Moonsammy, and M. Wisgerhof. 1967. Polyol accumulations in nervous tissue of rats with experimental diabetes and galactosaemia. J. Neurochem. 14: 10571066.

35. Eliasson, S. G. 1969. Properties of isolated nerve fibres from alloxanized rats. J. Neurol. Neurosurg. Psychiatry. 32: 525-529.

36. Kusama, H., and M. A. Stewart. 1970. Levels of myoinositol in normal and degenerating peripheral nerve. J. Neurochem. 17 : 317-323.

37. Broekhuyse, R. M. 1968. Changes in myo-inositol permeability in the lens due to cataractous conditions. Biochim. Biophys. Acta. 163: 269-272.

38. Kinoshita, J. H., G. W. Barber, L. O. Merola, and B. Tung. 1969. Changes in the levels of free amino acids and myo-inositol in the galactose-exposed lens. Invest. Ophthalmol. 8: 625-632.

39. Agranoff, B. W., J. A. Benjamin, and A. K. Hajra. 1969. Biosynthesis of phosphatidylinositol. Ann. N. Y. Acad. Sci. 165: 755-760.
40. Hawthorne, J. N. 1973. Phospholipid metabolism and transport of materials across the cell membrane. $B . B$. A. (Biochem. Biophys. Acta) Lib. 3: 423-440.

41. White, G. L., H. U. Schellhase, and J. N. Hawthorne. 1974. Phosphoinositide metabolism in rat superior cervical ganglion, vagus and phrenic nerve: effects of electrical stimulation and various blocking agents. J. Neurochem. 22 : 149-158.

42. Rerup, C. C. 1970. Drugs producing diabetes through damage of the insulin secreting cells. Pharmacol. Rev. 22 : 485-518.

43. Mauer, S. M., D. E. R. Sutherland, M. W. Steffes, R. J. Leonard, J. S. Najarian, A. F. Michael, and D. M. Brown. 1974. Pancreatic islet transplantation: effects on the glomerular lesion of experimental diabetes in the rat. Diabetes. 23 : 748-753.

44. Cameron, D. P., M. Amherdt, P. Leuenberger, L. Orci, and W. Stauffacher. 1973. Microvascular alterations in chronically streptozotocin-diabetic rats. Adv. Metab. Disord., Suppl. 2 : 257-269.

45. Greene, D. A., P. V. De Jesus, and A. I. Winegrad. 1974. Motor nerve conduction velocity in acute and chronic streptozotocin diabetes. Diabetes. 23: 357.

46. Dyck, P. J., W. J. Johnson, E. H. Lambert, and P. C. O'Brien. 1971. Segmental demyelination secondary to axonal degeneration in uremic neuropathy. Mayo Clin. Proc. 46: 400-431. 